\title{
Botanicals for photoprotection
}

\author{
Angeli E. Torres, Kevin M. Luk, Henry W. Lim \\ Photomedicine and Photobiology Unit, Department of Dermatology, Henry Ford Health System, Detroit, Michigan 48202, USA. \\ Correspondence to: Dr. Henry W. Lim, Department of Dermatology, Henry Ford Hospital, 3031 West Grand Boulevard, Detroit, \\ Michigan 48202, USA. E-mail: hlim1@hfhs.org \\ How to cite this article: Torres AE, Luk KM, Lim HW. Botanicals for photoprotection. Plast Aesthet Res 2020;7:57. \\ http://dx.doi.org/10.20517/2347-9264.2020.87 \\ Received: 20 Apr 2020 First Decision: 24 Aug 2020 Revised: 13 Sep 2020 Accepted: 29 Sep 2020 Published: 21 Oct 2020 \\ Academic Editor: Salvador Gonzalez Copy Editor: Cai-Hong Wang Production Editor: Jing Yu
}

\begin{abstract}
The importance of photoprotection against the deleterious effects of excessive and chronic exposure to sunlight is now well established. Photoprotective measures include behavioral modifications such as seeking shade, wearing photoprotective clothing, wide-brimmed hat and sunglasses, and applying sunscreen to exposed areas. Data on botanical topical and oral preparations have demonstrated photoprotective potential in in vitro, animal, and human studies. This review will focus on botanicals that have been most extensively studied, namely, Polypodium leucotomos extract, green tea, pomegranate, resveratrol, curcumin, and silymarin. These agents have shown promise in mitigating ultraviolet-induced acute changes on the skin, chronic photodamage, and even skin cancer prevention. However, it must be emphasized that current evidence indicates that these agents should be used as adjunctive measures rather than as a replacement of the photoprotective behavioral modifications described above.
\end{abstract}

Keywords: Botanical photoprotection, Polypodium leucotomos extract, green tea, pomegranate, resveratrol, curcumin, silymarin

\section{INTRODUCTION}

Electromagnetic radiation, including infrared, visible, and ultraviolet (UV) radiation (UVR), have both beneficial and harmful effects on the health of human skin. In particular, UVR exposure plays a significant role in the development of sunburns, photoaging, photoimmunosuppression, keratinocyte carcinomas, and cutaneous melanoma. They can also induce and exacerbate photosensitive dermatoses. Following 
exposure to UVR, reactive oxygen species (ROS) are generated in the skin, which result in oxidative stress. This contributes to acute UV-induced erythema (i.e., photoinflammation) and tanning through upregulation of cyclooxygenase-2 (COX-2), which is involved in early inflammation ${ }^{[1]}$. With chronic UVR exposure, the formation of photoproducts and ROS can lead to DNA damage, while at the same time cause downregulation of tumor suppressor genes. This allows cells to continue replicating while genetic mutations go unrepaired resulting in cancer formation (i.e., photocarcinogenesis) ${ }^{[1]}$. ROS in the skin can also activate proteins which play an important role in photoaging through blockage of collagen gene transcription, inhibition of collagen synthesis, and overexpression of enzymes that break down collagen ${ }^{[1]}$.

Photoprotection against UV and visible light is one preventative health strategy to reduce the negative effects of electromagnetic radiation. Historically, photoprotection has been achieved through topical routes. Photoprotection strategies include behavioral modifications such as seeking shade while outdoors, wearing photoprotective clothing including hats and sunglasses, as well as applying sunscreen on otherwise exposed skin sites ${ }^{[2,3]}$.

Sunscreens are the most widely recognized means of photoprotection by the public; however, they do have several limitations. These include the need for regular reapplication and lack of efficacy due to underapplication (i.e., not applying sufficient amounts). More recently, the ecological safety and potential human toxicity of organic sunscreens, such as oxybenzone and octinoxate, have raised concerns among dermatologists and the general public ${ }^{[3,4]}$. However, there is limited evidence of direct toxic effects of organic sunscreens in humans and coral reef species. Nevertheless, inorganic-based sunscreens - namely, zinc oxide and titanium oxide-based sunscreens - have been recommended as an alternative for those concerned about the potential health and environmental impact of organic sunscreens ${ }^{[4,5]}$. In addition, alternative photoprotective methods have gained increased interest as adjunct protection against UVR and visible light exposure.

Systemic photoprotection has been used in conjunction with topical photoprotection. It may be administered either subcutaneously ${ }^{[6]}$ or orally. Examples include vitamins, minerals, polyphenols, carotenoids, and $\alpha$-melanocyte stimulating hormone analog, as well as various plant-based agents that have been reported to yield photoprotective and anti-photocarcinogenic properties. These agents act through their antioxidant, anti-inflammatory, and immunomodulatory effects ${ }^{[7]}$. The focus of this article will be to review evidence-based systemic and topical botanicals as photoprotective agents.

\section{BOTANICALS WITH PHOTOPROTECTIVE PROPERTIES}

\section{Polypodium leucotomos extract}

Polypodium leucotomos is a tropical fern belonging to the family Polypodiaceae. It is native to Central and South America where it has been used traditionally to treat various skin diseases including psoriasis and atopic dermatitis ${ }^{[1,7,8]}$. At this time, Polypodium leucotomos extract (PLE) is the most well studied botanical photoprotective agent. It is commercially available worldwide as an over-the-counter oral supplement ${ }^{[\rho]}$. While there are many different preparations of PLE, most of the studies reported in peer-reviewed literature have been done with Fernblock ${ }^{\bullet}$ (Cantabria Labs, Madrid, Spain). PLE has antioxidant, anti-inflammatory, immunomodulatory, tumor suppressive, and anti-aging properties ${ }^{[1,7]}$. These qualities are mainly attributed to the fern's high polyphenol content, which is obtained from the leaves. Polyphenols are the most abundant class of antioxidants present in plant-based food and beverages. The polyphenols present in PLE are $p$-coumaric acid, chlorgenic acid, vanillic acid, caffeic acid, and ferulic acid. Of these, the most powerful antioxidants are ferulic and caffeic acids ${ }^{[1]}$. It must be emphasized that the concentration of these constituents can vary depending on the PLE preparation. Accordingly, the different PLE preparations may vary in term of their photoprotective ability. This was demonstrated in a study by Gonzalez et al. ${ }^{[10]}$, where the photoprotective activity of six different PLE preparations (including Fernblock ${ }^{\circledR}$ ) were tested in vitro. 
Results showed that Fernblock ${ }^{\oplus}$ was by far the most active ( $>5 \mathrm{x}$ on fibroblasts and $>3 \mathrm{x}$ on keratinocytes) compared to the other five PLE preparations, of which two were found to have almost no activity ${ }^{[10]}$.

PLE augments the body's natural antioxidant system and minimizes the accumulation of ROS in the skin. It has the unique ability to scavenge superoxide anion in contrast to conventional antioxidants, which can neutralize singlet oxygen only ${ }^{[7]}$. As an anti-inflammatory compound, PLE has been shown to suppress UV-induced erythema and reduce cutaneous phototoxicity from photochemotherapy ${ }^{[7]}$. It can also increase the minimal erythema dose (MED), the UVR dose required for immediate pigment darkening, and the minimal phototoxic UVR dose $e^{[9]}$. One randomized controlled trial found that healthy human subjects who took oral PLE supplements $240 \mathrm{mg}$ twice daily for 60 days were 6 times less likely to have a sunburn, 22 times more likely to have increased MED, and 15 times less likely to exhibit visible erythema postUVB exposure ${ }^{[11]}$. These effects were quantified in a later study by Kohli et al. ${ }^{[12]}$ where it was noted that the intensity of UVB-induced erythema decreased by an average of $8 \%$ post-PLE supplementation. Similarly, one study which did not utilize Fernblock ${ }^{\bullet}$, noted increased MED among subjects with skin phototype I-III following once daily intake of an oral supplement containing PLE for 12 weeks ${ }^{[13]}$. The possible mechanism underlying PLE's anti-inflammatory properties include the inhibition of transcription factors and cytokines that mediate photoinflammation, namely, tumor necrosis factor (TNF), inducible nitric oxide synthase (iNOS), activator protein 1 (AP-1), and nuclear factor kappa B (NF- $\kappa \mathrm{B})$. In addition, PLE can also reduce the expression of COX-2 and prostaglandin E2, both of which are involved in the initial steps of the inflammatory pathway ${ }^{[1,7]}$.

In terms of immunomodulation, PLE has been found to preserve epidermal Langerhans cells that are otherwise depleted as a result of $\mathrm{UVR}^{[7]}$. Its anti-tumor effects were exhibited in mice that were given oral PLE daily as evidenced by increased expression of the tumor suppressor protein $\mathrm{p} 53^{[14]}$. PLE has likewise been shown to accelerate extracellular matrix turnover and promote renewal of dermal collagen through inhibition of matrix metalloproteinases (MMPs) and upregulation of tissue inhibitor of metalloproteinase, thereby supporting its role in the prevention of photoaging ${ }^{[1,8]}$.

PLE can also provide photoprotection from wavelengths beyond UV. In a study by Mohammad et al. ${ }^{[15]}$, patients taking $480 \mathrm{mg}$ of PLE daily demonstrated a substantial decrease in visible light-induced persistent pigment darkening and delayed tanning. Furthermore, an in vitro study by Zamarrón et al. ${ }^{[16]}$ showed that PLE decreased cell death and collagen degradation in human dermal fibroblasts that were exposed to visible light and near infrared radiation. Additional benefits of PLE include suppression of photodermatoses such as polymorphous light eruption and solar urticaria. It can also be used as an adjunctive treatment for vitiligo and actinic keratosis in combination with phototherapy and photodynamic therapy, respectively ${ }^{[14]}$.

PLE has relatively minor side effects, including mild to moderate gastrointestinal symptoms and pruritus, which have been reported in a small percentage of patients receiving doses ranging from $120 \mathrm{mg}$ to $1,080 \mathrm{mg}$ daily ${ }^{[17]}$. No changes in physical examination, vital signs, and laboratory tests (complete metabolic panel and clotting studies) were observed from baseline among patients taking $480 \mathrm{mg}$ daily of PLE for 2 months ${ }^{[11]}$.

\section{Green tea}

Produced from the leaves and leaf buds of Camellia sinensis, green tea is one of the most widely consumed beverages in the world. Its many benefits are primarily due to its polyphenols - otherwise known as green tea catechins (GTC) - of which the most abundant (65\%) is epigallocatechin-3-gallate ${ }^{[7,8,14]}$. Similar to other phenolic compounds, GTC has antioxidant, anti-inflammatory, immunomodulatory, and chemopreventive properties.

In vitro studies on human keratinocytes have shown that GTCs can inhibit activation of AP-1 and NF- $\mathrm{B}$, decrease UVB-induced apoptosis, and stimulate the production of interleukin (IL)-12. IL-12 is postulated 
to play a role in photoprotection since photoinflammation, impaired DNA repair, and tumorigenesis are associated with an absence of IL-12. In mice, topical and oral GTC has been found to protect against photoinflammation and photocarcinogenesis through inhibition of the mitogen-activated protein kinase inflammatory pathway and upregulation of genes involved in DNA repair.

In humans, previous studies have reported that topical GTC reduces photodamage by decreasing production of UVR-induced cyclobutane pyrimidine dimers (CPDs) and visible sunburn ${ }^{[18,19]}$. However, GTCs have poor skin penetration when topically applied due to their poor lipid solubility ${ }^{[7]}$. They are also subject to photodegradation. ${ }^{[19]}$ In contrast, orally administered GTC has been shown to have good skin bioavailability as evidenced by their presence in skin biopsy specimens and skin blister fluid. This was noted after GTC supplementation equivalent to 5 cups of green tea daily for 12 weeks ${ }^{[20]}$. However, there is conflicting evidence in the literature as to the efficacy of oral GTC as a photoprotectant ${ }^{[21,22]}$.

One study showed that women who consumed one liter of green tea daily for 3 months were found to have increased skin elasticity, improved skin texture, decreased transepidermal water loss, and increased cutaneous blood flow ${ }^{[22]}$. In contrast, a study of healthy, light-skinned (skin phototype I-II) adults revealed no significant differences in UVR-induced erythema, dermal leukocytic infiltration, and induction of cyclooxygenase and lipoxygenase inflammatory pathways among those taking daily GTC supplements $\left(1,080 \mathrm{mg}\right.$ plus $100 \mathrm{mg}$ vitamin C) compared to those in the placebo group ${ }^{[21]}$. Further studies are needed to reconcile these conflicting evidences.

\section{Pomegranate}

The extract from Punica granatum or pomegranate is rich in phenolic compounds, specifically, anthocyanins, catechins, and tannins ${ }^{[7]}$. Significant amounts of these compounds are present in different parts of the fruit but are most concentrated in the peel and juice ${ }^{[23]}$. At present, pomegranate extract is widely available as an over-the-counter oral supplement or topical formulation, and is often incorporated in commercially sold skin care products.

Pomegranate extract has anti-inflammatory properties and a very potent antioxidant activity - even greater than that of green tea or red wine ${ }^{[7,8]}$. It confers photoprotection through inhibition of UV-induced production of free radicals, erythema and burning, DNA damage, cell proliferation, and apoptosis ${ }^{[8,24]}$. It can also decrease collagen breakdown ${ }^{[25]}$.

Murine in vivo studies have demonstrated that topical application of pomegranate extract can replenish antioxidants (including catalase, peroxidase, and superoxide dismutase), as well as reduce photoinflammation. This was evidenced by decreased skin edema, epidermal thickening, dermal neutrophilic infiltrates, ornithine decarboxylase, and COX-2. Additionally, topical pomegranate extract has been found to prolong the latency and lessen the multiplicity of skin tumors, thereby supporting its benefit against photocarcinogenesis ${ }^{[26]}$.

Oral administration of pomegranate extract was found to be protective against UVB-induced skin cancer formation in mice through downregulation of COX-2, iNOS, cyclin D2, and MMPs. Women who consumed $1000 \mathrm{mg}$ of pomegranate extract or 8 ounces of pomegranate juice daily for 12 weeks were reported to have increased MED in a randomized controlled, open-label study ${ }^{[25]}$.

The anti-aging benefits of pomegranate extract were further investigated in a 2017 study by Kang et al. ${ }^{[2]}$ using orally administered pomegranate juice concentrated powder (PCP) in hairless mice. In this 15-week, placebo-controlled trial, PCP was given at 100, 200, or $400 \mathrm{mg} / \mathrm{kg}$ once daily, and 1 hour prior to thrice weekly UVB exposure. The investigators reported dose-dependent decreases in wrinkle formation, skin 
edema, expression of pro-inflammatory cytokines, apoptosis, and MMP activity. Moreover, PCP-treated mice showed increases in water, collagen type I, and hyaluronic acid contents in the skin, indicating a skin moisturizing effect.

\section{Resveratrol (Grape seed, grape peel, and red wine)}

Resveratrol is a stilbenoid compound belonging to the non-flavonoid class of polyphenols ${ }^{[28]}$. It is present in grape seeds, grape peels, as well as red wine ${ }^{[7]}$. In terms of photoprotection, resveratrol has demonstrated anti-oxidant, anti-inflammatory, and anti-tumor effects in several in vitro, animal, and human studies ${ }^{[8]}$.

In an in vitro study by Zhou et al. ${ }^{[29]}$, pre-treatment of cultured human keratinocytes with different concentrations of resveratrol prior to UVB irradiation resulted in concentration-dependent increase in cell viability and a decreased rate of apoptosis. Following UVB irradiation, samples that were pre-treated with resveratrol had 15\%-52\% more viable cells and 15\%-22\% less apoptosis than non-treated samples. Furthermore, resveratrol pre-treated samples demonstrated a 1.4-fold increase in expression of Bcl-2 (antiapoptotic protein), and decreased expression of Bax and caspase-3 (i.e., pro-apoptotic proteins) by $52 \%$ and $45 \%$, respectively.

In mice, topical application of resveratrol prior to UVB radiation exposure was found to inhibit UVBinduced skin edema, inflammation, generation of ROS (e.g., hydrogen peroxide and lipoperoxides), and induction of COX and ornithine carboxylase ${ }^{[8]}$. Meanwhile, oral administration demonstrated antitumor effect through alteration of tumor growth factor beta and NF- $\kappa \mathrm{B}$, both of which are involved in cell proliferation and tumorigenesis ${ }^{[1,7]}$.

In humans, resveratrol has been found to afford partial protection against UVR-induced photodamage. $\mathrm{Wu}$ et al. ${ }^{[30]}$ conducted a study on 15 healthy volunteers who were subject to repetitive UVR exposure from a solar simulator at a dose of 1.5 MED. Results showed that skin sites which were treated with topical resveratrol had less UV-induced erythema and sunburn cell formation compared to placebo (vehicle only) or negative control (no treatment).

\section{Curcumin (Turmeric)}

Curcumin is the active constituent of turmeric (Curcuma longa), a rhizomatous plant native to South Asia that is now grown in many tropical and subtropical regions worldwide. Turmeric is commonly used as a spice, coloring agent, and for various indications in Ayurvedic and traditional Chinese medicine ${ }^{[8,14]}$.

Previous in vitro studies utilizing human keratinocytes and epidermoid carcinoma cells (i.e., squamous cell carcinoma cell line) have found that curcumin decreases UVB-induced apoptosis and inflammation through inhibition of the NF- $\mathrm{KB}$ and MAPK pathways ${ }^{[8,14]}$. In keratinocytes and fibroblasts, curcumin decreases the expression of MMP-1, which may help reduce the appearance of wrinkles in photoaged $\operatorname{skin}^{[8]}$. Moreover, curcumin has demonstrated ability to decrease squamous cell carcinoma tumor growth in mice ${ }^{[14]}$.

Curcumin may also confer protection against UVA-mediated photodamage. According to a study by Liu et al. ${ }^{[31]}$ (2018), pre-treatment with curcumin 2 h prior to UVA exposure prevented accumulation of ROS and restores the innate antioxidant function of human fibroblasts in vitro. In addition, curcumin was shown to mitigate UVA-induced apoptosis, inflammation, and collagen degradation.

\section{Silymarin (Milk thistle)}

Silymarin is an isoflavone derived from the seeds of the milk thistle plant (Silybum marianum), which is one of the oldest known medicinal herbs used in traditional European medicine. Nowadays, it is 
Table 1. Most extensively studied botanicals for photoprotection

\begin{tabular}{|c|c|c|c|c|}
\hline Botanical agent & Spectrum & Mechanism of photoprotection & Routes & Models \\
\hline $\begin{array}{l}\text { Polypodium } \\
\text { leucotomos extract }\end{array}$ & $\begin{array}{l}\text { UVB, } V L+ \\
\text { UVA1, IR-A }\end{array}$ & $\begin{array}{l}\text { Antioxidant: Augments natural antioxidant system, can scavenge } \\
\text { superoxide anion } \\
\text { Anti-inflammatory: Increases MED, dose required for IPD, and } \\
\text { minimal phototoxic dose; inhibits proinflammatory transcription } \\
\text { factors, mediators, and cytokines; decreases VL + UVA1 induced } \\
\text { PPD and DT } \\
\text { Immunomodulatory: Preserves eLCs } \\
\text { Anti-tumor: Increases expression of p53 tumor suppressor gene } \\
\text { Anti-aging: Downregulates MMP; upregulates TIMP; prevents VL } \\
\text { and IR-A induced cell death and collagen degradation } \\
\text { Other: Suppresses photodermatoses }\end{array}$ & Oral & $\begin{array}{l}\text { In vitro, mouse, } \\
\text { human }\end{array}$ \\
\hline Green tea & UVB & $\begin{array}{l}\text { Increases skin elasticity and blood flow; stimulates IL-12; decreases } \\
\text { apoptosis, CPDs, sunburn, and TEWL; inhibits AP-1, NFKB, MAPK }\end{array}$ & Topical, oral & $\begin{array}{l}\text { In vitro, mouse, } \\
\text { human }\end{array}$ \\
\hline Pomegranate & UVB & $\begin{array}{l}\text { Increases MED, skin moisture, and tumor latency; decreases } \\
\text { inflammation and multiplicity of tumors; inhibits ROS, erythema, } \\
\text { burning, DNA damage, cell proliferation, apoptosis, and collagen } \\
\text { breakdown }\end{array}$ & Topical, oral & Mouse, human \\
\hline $\begin{array}{l}\text { Resveratrol } \\
\text { (Grape seed, grape } \\
\text { peel, and red wine) }\end{array}$ & UVB & $\begin{array}{l}\text { Increases cell viability; decreases apoptosis, erythema, and } \\
\text { sunburn cell formation; inhibits COX, ornithine carboxylase, TGF } \\
\text { beta, and NF- } \kappa \text { B }\end{array}$ & Topical, oral & $\begin{array}{l}\text { In vitro, mouse, } \\
\text { human }\end{array}$ \\
\hline $\begin{array}{l}\text { Curcumin } \\
\text { (Turmeric) }\end{array}$ & UVA, UVB & $\begin{array}{l}\text { Prevents ROS accumulation; decreases apoptosis, inflammation, } \\
\text { MMP-1 expression, and SCC tumor growth; inhibits NF- } \kappa B \text { and } \\
\text { MAPK }\end{array}$ & Topical, oral & $\begin{array}{l}\text { In vitro, mouse, } \\
\text { human }\end{array}$ \\
\hline $\begin{array}{l}\text { Silymarin } \\
\text { (Milk Thistle) }\end{array}$ & UVA, UVB & $\begin{array}{l}\text { Activates p53 tumor suppressor gene; decreases MMP-1 } \\
\text { activation, inflammation, ROS, DNA damage, apoptosis, IL-10, and } \\
\text { CPDs; inhibits collagenase, hyaluronidase, and elastase }\end{array}$ & NA & In vitro \\
\hline
\end{tabular}

UVB: ultraviolet B; UVA: ultraviolet A; VL + UVA1: visible light + ultraviolet A1; IR-A: infrared A; MED: minimal erythema dose; IPD: immediate pigment darkening; PPD: persistent pigment darkening; DT: delayed tanning; eLCs: epidermal Langerhans cells; MMP: matrix metalloproteinase; TIMP: tissue inhibitor of metalloproteinase; IL: interleukin; CPD: cyclobutane pyrimidine dimers; TEWL: transepidermal water loss; AP-1: activator protein 1; NFKB: nuclear factor kappa B; MAPK: mitogen-activated protein kinase; ROS: reactive oxygen species; DNA: deoxyribonucleic acid; COX: cyclooxygenase; TGF: tumor growth factor; SCC: squamous cell carcinoma; NA: not applicable

chiefly marketed as an oral hepatic supplement and has been used to treat hepatitis, alcoholic liver diseases, cirrhosis, and toxin-induced hepatotoxicity. The polyphenols present in silymarin, which is more appropriately termed flavonolignans, are silybin, silychristin, silydianin, isosilybin, and 2,3-dehydrosilybin ${ }^{[32-34]}$.

The antioxidant and anti-inflammatory effects of silymarin are similar to other phenolic compounds in that it downregulates the UVB-induced generation of ROS, expression of inflammatory transcription factors and cytokines (e.g., TNF, IL-1, and iNOS), and activation of inflammatory pathways including COX and lipoxygenase pathways. As an immunomodulatory compound, silymarin reverses UVB-induced immunosuppression through decreased production of IL-10, which has been found to be elevated in the presence of cancers and are thought to be responsible for the cancer's ability to evade the host's immune response. In addition, silymarin imparts protection against UVB photocarcinogenesis through activation of p53, and reduction of pyrimidine photoproduct formation ${ }^{[34]}$.

In terms of protection against UVA, one in vitro study on human dermal fibroblasts revealed that pretreatment with silymarin one hour prior to UVA exposure decreased ROS production, DNA strand breaks, activation of MMP-1, and the pro-apoptotic protein caspase- $3^{[35]}$. In another in vitro study, silymarin and its flavonolignans were found to inhibit the UVA-induced activity of collagenase, hyaluronidase, and elastase, which are respectively responsible for skin wrinkling, loss of hydration, and sagging, indicating an antiphotoaging effect ${ }^{[33]}$.

However, a study by Fidrus et al. ${ }^{[36]}$ (2019) showed contradictory results wherein silymarin pre-treatment of human keratinocytes in vitro 30 min prior to UVA exposure enhanced UVA-induced cytotoxicity in a dose 
Table 2. Other botanicals with photoprotective effects

\begin{tabular}{|c|c|c|c|c|c|}
\hline Botanical agent & Spectrum & Mechanism of photoprotection & Routes & Models & Ref. \\
\hline Cocoa extract & UVB & $\begin{array}{l}\text { Increases MED; decreases erythema and } \\
\text { skin wrinkling }\end{array}$ & Oral & Mouse, Human & {$[7]$} \\
\hline $\begin{array}{l}\text { Rosemary plus grapefruit } \\
\text { extract }\end{array}$ & UVA, UVB & $\begin{array}{l}\text { Increases MED, skin elasticity; decreases } \\
\text { erythema, lipoperoxides, and skin wrinkling }\end{array}$ & Oral & Human & [37] \\
\hline Strawberry extract & UVA & Increases cell viability & NA & In vitro & [38] \\
\hline Blueberry extract & $\begin{array}{l}\text { UVA, UVB and } \\
\text { UVC }\end{array}$ & Increases cell viability & NA & In vitro & [39] \\
\hline Melon concentrate & UVA, UVB & $\begin{array}{l}\text { Increases MED and endogenous antioxidants; } \\
\text { decreases sunburn cells }\end{array}$ & Topical, oral & Human, In vitro & [40] \\
\hline $\begin{array}{l}\text { Sechium edule (chayote) } \\
\text { extract }\end{array}$ & UVA & $\begin{array}{l}\text { Increases DNA repair; decreases apoptosis, } \\
\text { ROS, DNA damage, and CPDs }\end{array}$ & NA & In vitro & [41] \\
\hline $\begin{array}{l}\text { Oenanthe javanica (water } \\
\text { celery) extract }\end{array}$ & UVB & $\begin{array}{l}\text { Increases collagen type I and III; decreases } \\
\text { MMP-1, MMP-3, TNF, and COX-2 expression }\end{array}$ & Topical & Mouse & [42] \\
\hline
\end{tabular}

UVB: ultraviolet B; UVA: ultraviolet A; UVC: ultraviolet C; MMP: matrix metalloproteinase; TNF: tumor necrosis factor; COX-2: cyclooxygenase 2; MED: minimal erythema dose; DNA: deoxyribonucleic acid; ROS: reactive oxygen species; CPD: cyclobutane pyrimidine dimers; NA: Not applicable

dependent manner (i.e., less viable cells with higher silymarin doses). In addition, silymarin pre-treated keratinocytes produced higher amounts of CPDs following UVA exposure compared to non-pre-treated keratinocytes. The mechanism for this silymarin-induced phototoxicity is still poorly understood.

Table 1 summarizes the mechanism of photoprotection and spectrum coverage of the botanical agents discussed above.

\section{Others}

Other botanicals that have been reported to have photoprotective effects, albeit have not been as rigorously studied, are summarized in Table 2.

\section{CONCLUSION}

Botanical-based photoprotection is likely to increase in popularity as consumer trends worldwide continue to place an emphasis on naturally occurring compounds used solely or in conjunction with synthetic products. The botanicals reviewed above currently have the most evidence available and can serve as options for providers to recommend to patients. These oral and topical botanical products act through a variety of biologic mechanisms to confer protection against the adverse effects of UVR. However, unlike sunscreens, botanical products are not subject to FDA regulations and so rigorous efficacy and safety testing through large-scale controlled therapeutic trials are lacking for many of these agents. As such, their true photoprotective benefit compared to established measures like seeking shade, donning UV-blocking garments, or organic or inorganic topical sunscreens remains to be verified. In addition, the stability of botanical ingredients as well as the optimal concentration of their constituents is unregulated. Therefore, while evidence on their use as an adjunctive means of photoprotection appears favorable, they should be used in conjunction with, and not as a replacement of, pre-existing photoprotection recommendations. Finally, as the biologic effects of other wavelengths of electromagnetic radiation such as visible and infrared ranges continue to be elucidated, it will be critical for future research to evaluate the potential applicability of botanicals for protection in that realm as well.

\section{DECLARATIONS}

\section{Authors' contributions}

Made substantial contributions to literature search and writing of initial manuscript: Torres AE, Luk KM Contributed to writing and editing of manuscript: Lim HW 


\section{Availability of data and materials}

Not applicable.

\section{Financial support and sponsorship}

None.

\section{Conflicts of interest}

Torres AE and Luk KM declared that there are no conflicts of interest; Lim HW is an investigator for Incyte, L'Oréal, Pfizer, PCORI, has served as consultant for Pierre Fabre, ISDIN, Ferndale, and Galderma, and has participated as a speaker in general educational session for Johnson \& Johnson, and Ra Medical System.

\section{Ethical approval and consent to participate}

Not applicable.

\section{Consent for publication}

Not applicable.

\section{Copyright}

(c) The Author(s) 2020.

\section{REFERENCES}

1. Parrado C, Mascaraque M, Gilaberte Y, Juarranz A, Gonzalez S. Fernblock (Polypodium leucotomos extract): molecular mechanisms and pleiotropic effects in light-related skin conditions, photoaging and skin cancers, a review. Int J Mol Sci 2016;17:1026.

2. Krutmann J, Passeron T, Gilaberte Y, Granger C, Leone G, et al. Photoprotection of the future: challenges and opportunities. J Eur Acad Dermatol Venereol 2020;34:447-54.

3. Yeager DG, Lim HW. What's new in photoprotection: a review of new concepts and controversies. Dermatol Clin 2019;37:149-57.

4. Schneider SL, Lim HW. Review of environmental effects of oxybenzone and other sunscreen active ingredients. J Am Acad Dermatol 2019;80:266-71.

5. Schneider SL, Lim HW. A review of inorganic UV filters zinc oxide and titanium dioxide. Photodermatol Photoimmunol Photomed 2019;35:442-6.

6. Langendonk JG, Balwani M, Anderson KE, Bonkovsky HL, Anstey AV, et al. Afamelanotide for erythropoietic protoporphyria. N Engl J Med 2015;373:48-59.

7. Parrado C, Philips N, Gilaberte Y, Juarranz A, González S. Oral photoprotection: effective agents and potential candidates. Front Med (Lausanne) 2018;5:188

8. Saewan N, Jimtaisong A. Natural products as photoprotection. J Cosmet Dermatol 2015;14:47-63.

9. Lim HW, Arellano-Mendoza MI, Stengel F. Current challenges in photoprotection. J Am Acad Dermatol 2017;76:S91-9.

10. Gonzalez S, Lucena SR, Delgado P, Juarranz A. Comparison of several hydrophilic extracts of Polypodium leucotomos reveals different antioxidant moieties and photoprotective effects in vitro. J Med Plants Res 2018;12:336-45.

11. Nestor MS, Berman B, Swenson N. Safety and efficacy of oral Polypodium leucotomos extract in healthy adult subjects. J Clin Aesthet Dermatol 2015;8:19-23.

12. Kohli I, Shafi R, Isedeh P, Griffith JL, Al-Jamal MS, et al. The impact of oral Polypodium leucotomos extract on ultraviolet B response: a human clinical study. J Am Acad Dermatol 2017;77:33-41.e1.

13. Granger C, Aladren S, Delgado J, Garre A, Trullas C, et al. Prospective evaluation of the efficacy of a food supplement in increasing photoprotection and improving selective markers related to skin photo-ageing. Dermatol Ther (Heidelb) 2020;10:163-78.

14. Rabinovich L, Kazlouskaya V. Herbal sun protection agents: Human studies. Clin Dermatol 2018;36:369-75.

15. Mohammad TF, Kohli I, Nicholson CL, Treyger G, Chaowattanapanit S, et al. Oral Polypodium leucotomos extract and its impact on visible light-induced pigmentation in human subjects. J Drugs Dermatol 2019;18:1198-203.

16. Zamarrón A, Lorrio S, González S, Juarranz Á. Fernblock prevents dermal cell damage induced by visible and infrared A radiation. Int J Mol Sci 2018;19:2250.

17. Winkelmann RR, Del Rosso J, Rigel DS. Polypodium leucotomos extract: a status report on clinical efficacy and safety. J Drugs Dermatol 2015;14:254-61.

18. Farrar MD, Huq R, Mason S, Nicolaou A, Clarke KA, et al. Oral green tea catechins do not provide photoprotection from direct DNA damage induced by higher dose solar simulated radiation: a randomized controlled trial. J Am Acad Dermatol 2018;78:414-6.

19. Nwanodi O. Skin protective nutraceuticals: the current evidence in brief. Healthcare (Basel) 2018;6:40. 
20. Clarke KA, Dew TP, Watson RE, Farrar MD, Osman JE, et al. Green tea catechins and their metabolites in human skin before and after exposure to ultraviolet radiation. J Nutr Biochem 2016;27:203-10.

21. Farrar MD, Nicolaou A, Clarke KA, Mason S, Massey KA, et al. A randomized controlled trial of green tea catechins in protection against ultraviolet radiation-induced cutaneous inflammation. Am J Clin Nutr 2015;102:608-15.

22. Heinrich U, Moore CE, De Spirt S, Tronnier H, Stahl W. Green tea polyphenols provide photoprotection, increase microcirculation, and modulate skin properties of women. J Nutr 2011;141:1202-8.

23. Baccarin T, Mitjans M, Ramos D, Lemos-Senna E, Vinardell MP. Photoprotection by Punica granatum seed oil nanoemulsion entrapping polyphenol-rich ethyl acetate fraction against UVB-induced DNA damage in human keratinocyte (HaCaT) cell line. J Photochem Photobiol B 2015;153:127-36.

24. Baccarin T, Mitjans M, Lemos-Senna E, Vinardell MP. Protection against oxidative damage in human erythrocytes and preliminary photosafety assessment of Punica granatum seed oil nanoemulsions entrapping polyphenol-rich ethyl acetate fraction. Toxicol In Vitro 2015;30:421-8.

25. Henning SM, Yang J, Lee RP, Huang J, Hsu M, et al. Pomegranate juice and extract consumption increases the resistance to UVBinduced erythema and changes the skin microbiome in healthy women: a randomized controlled trial. Sci Rep 2019;9:14528.

26. Mintie CA, Singh CK, Ahmad N. Whole fruit phytochemicals combating skin damage and carcinogenesis. Transl Oncol 2020;13:146-56.

27. Kang SJ, Choi BR, Kim SH, Yi HY, Park HR, et al. Beneficial effects of dried pomegranate juice concentrated powder on ultraviolet B-induced skin photoaging in hairless mice. Exp Ther Med 2017;14:1023-36.

28. de Lima Cherubim DJ, Buzanello Martins CV, Oliveira Fariña L, da Silva de Lucca RA. Polyphenols as natural antioxidants in cosmetics applications. J Cosmet Dermatol 2020;19:33-7.

29. Zhou F, Huang X, Pan Y, Cao D, Liu C, et al. Resveratrol protects HaCaT cells from ultraviolet B-induced photoaging via upregulation of HSP27 and modulation of mitochondrial caspase-dependent apoptotic pathway. Biochem Biophys Res Commun 2018;499:662-8.

30. Wu Y, Jia LL, Zheng YN, Xu XG, Luo YJ, et al. Resveratrate protects human skin from damage due to repetitive ultraviolet irradiation. J Eur Acad Dermatol Venereol 2013;27:345-50.

31. Liu X, Zhang R, Shi H, Li X, Li Y, et al. Protective effect of curcumin against ultraviolet A irradiation-induced photoaging in human dermal fibroblasts. Mol Med Rep 2018;17:7227-37.

32. Skarupova D, Vostalova J, Rajnochova Svobodova A. Ultraviolet A protective potential of plant extracts and phytochemicals. Biomed Pap Med Fac Univ Palacky Olomouc Czech Repub 2020;164:1-22.

33. Vostálová J, Tinková E, Biedermann D, Kosina P, Ulrichová J, et al. Skin protective activity of silymarin and its flavonolignans. Molecules 2019;24:1022.

34. Dorjay K, Arif T, Adil M. Silymarin: an interesting modality in dermatological therapeutics. Indian J Dermatol Venereol Leprol 2018;84:238-43.

35. Rajnochová Svobodová A, Gabrielová E, Michaelides L, Kosina P, Ryšavá A, et al. UVA-photoprotective potential of silymarin and silybin. Arch Dermatol Res 2018;310:413-24.

36. Fidrus E, Ujhelyi Z, Fehér P, Hegedűs C, Janka EA, et al. Silymarin: friend or foe of UV exposed keratinocytes? Molecules 2019;24:1652.

37. Nobile V, Michelotti A, Cestone E, Caturla N, Castillo J, et al. Skin photoprotective and antiageing effects of a combination of rosemary (Rosmarinus officinalis) and grapefruit (Citrus paradisi) polyphenols. Food Nutr Res 2016;60:31871.

38. Gasparrini M, Forbes-Hernandez TY, Afrin S, Alvarez-Suarez JM, Gonzàlez-Paramàs AM, et al. A pilot study of the photoprotective effects of strawberry-based cosmetic formulations on human dermal fibroblasts. Int J Mol Sci 2015;16:17870-84.

39. Bucci P, Prieto MJ, Milla L, Calienni MN, Martinez L, et al. Skin penetration and UV-damage prevention by nanoberries. J Cosmet Dermatol 2018;17:889-99.

40. Egoumenides L, Gauthier A, Barial S, Saby M, Orechenkoff C, et al. A specific melon concentrate exhibits photoprotective effects from antioxidant activity in healthy adults. Nutrients 2018;10:437.

41. Metral E, Rachidi W, Damour O, Demarne F, Bechetoille N. Long-term genoprotection effect of Sechium edule fruit extract against UVA irradiation in keratinocytes. Photochem Photobiol 2018;94:343-50.

42. Her Y, Shin BN, Lee YL, Park JH, Kim DW, et al. Oenanthe javanica extract protects mouse skin from UVB radiation via attenuating collagen disruption and inflammation. Int J Mol Sci 2019;20:1435. 